\title{
Calculation of Fundamental Groups via Computational Paths
}

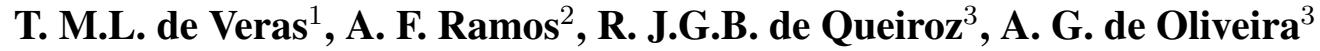 \\ ${ }^{1}$ Departamento de Matemática, Universidade Federal Rural de Pernambuco \\ ${ }^{2}$ Microsoft Redmond, USA \\ ${ }^{3}$ Centro de Informática, Universidade Federal de Pernambuco \\ tiago.veras@ufrpe.br arfreita@microsoft.com \{ruy,ago\}@cin.ufpe.br
}

\begin{abstract}
We address the question as to how to formalise the concept of computational paths (sequences of rewrites) as equalities between two terms of the same type. The intention is to demonstrate the use of a term rewriting system in performing computations with these computational paths, establishing equalities between equalities, and further higher equalities, in particular, in the calculation of fundamental groups of surfaces such as the circle, the torus and the real projective plane.
\end{abstract}

\section{Introduction}

The identity type is arguably one of the most interesting entities of Martin-Löf type theory. From any type $A$, one can construct the identity type $I d_{A}(x, y)$ as the relation of identity between two terms of $A$, i.e., if there is $x={ }_{p} y: A$, where $p$ is a witness or proof that $x$ is indeed equal to $y$. And this allows for the interpretation of the identity type as the type of paths between two points of the same space giving rise to the interesting rendition of equality as a collection of homotopical paths. This connection of type theory and homotopy theory makes type theory a suitable foundation for both computation and mathematics. Nevertheless, in the 'oficial' formulation of homotopy type theory this interpretation is only a semantical one since paths do not have a syntactical counterpart. For that reason, the addition of terms to formalise paths in the syntax of what might be called 'explicit' homotopy type theory has been proposed by de Queiroz, Ramos and de Oliveira [de Queiroz et al. 2016, Ramos et al. 2017]. The idea is to make use of a calculus of terms representing computational paths, proposed by de Queiroz and Gabbay in 1994 [de Queiroz and Gabbay 1994], and demonstrate that it can be useful for formalizing the identity type in a more explicit manner. And this is shown to be useful in calculating and formally proving the fundamental group of surfaces.

On the other hand, one of the main interesting points of the interpretation of logical connectives via deductive systems which use a labelling system is the clear separation between a functional calculus on the labels (the names that record the steps of the proof) and a logical calculus on the formulas [Martin-Löf 1975, de Queiroz and Gabbay 1994]. The works of [de Queiroz et al. 2016, de Queiroz and Gabbay 1994, de Queiroz et al. 2011] claim that the harmony that comes with this separation makes labelled natural deduction a suitable framework to study and develop a theory of equality for natural deduction. Take, for example, the following cases from the $\lambda$-calculus :

$$
(\lambda x .(\lambda y . y x)(\lambda w . z w)) v \triangleright_{\eta}(\lambda x .(\lambda y . y x) z) v \triangleright_{\beta}(\lambda y . y v) z \triangleright_{\beta} z v
$$




$$
(\lambda x .(\lambda y . y x)(\lambda w . z w)) v \triangleright_{\beta}(\lambda x(\lambda w . z w) x) v \triangleright_{\eta}(\lambda x . z x) v \triangleright_{\beta} z v
$$

In the theory of the $\beta \eta$-equality of $\lambda$-calculus, we can indeed say that $(\lambda x .(\lambda y . y x)(\lambda w . z w)) v$ is equal to $z v$. Moreover, there are at least two ways of obtaining these equalities. We can go further, and call $s$ the first sequence of rewrites that establish that $(\lambda x .(\lambda y . y x)(\lambda w . z w)) v$ is indeed equal to $z v$. The second one, for example, can be called $r$. Thus, we can say that this equality is established by $s$ and $r$. As such, $s$ and $r$ are examples of an entity here called computational path.

Since we now have terms (that establish the equality between two (path) terms, interesting questions arise: (i) is $s$ different from $r$; (ii) are there normal forms of this equality proof; (iii) if $s$ is equal to $r$, how can we prove this? Questions like these can be answered when we work in a labelled natural deduction framework. The idea is that we are not limited by the calculus on the formulas, but we can also define and work with rules that apply to the labels. That way, we can use these rules to formally establish the equality between these labels, i.e., establish equalities between equalities. Here we shall use a system proposed by [de Oliveira 1995] and known as $L N D_{E Q^{-}} T R S$.

A recent presentation by Michael Shulman (Homotopy type theory - A high-level language for invariant mathematics, March 2019) draws attention to the importance of Voevodsky's homotopy type theory in finding a proper framework to deal with higher-order equalities: "Homotopy type theory is a high-level abstract framework for working with sameness."

In that context, the intention here is show how to concretely witness an interesting connection: to use a labelled natural deduction system together with a rewriting system $L N D_{E Q}-T R S$ to obtain topological results about fundamental groups. Indeed, the idea is to develop a framework for dealing with such expressions representing computational paths and show that it is powerful enough to provide the formal tools to calculate the fundamental group of surfaces such as the circle, the torus and the real projective plane. Taking into account that in mathematics the calculation of a fundamental group is quite laborious, we believe our framework may prove useful in this calculation in a less complex form. Nevertheless, to obtain this result we first need to formally define the concept of computational paths and define $L N D_{E Q^{-}} T R S$.

This is part of an ongoing project [Ramos et al. 2017, Veras et al. 2019, Veras et al. 2020] which, while it looks for the use of homotopy structures such as groupoids in the study of semantics of computation, it also seeks to demonstrate the utility and the impact of the so-called Curry-Howard interpretation of logical deduction in the actual practice of an important area of mathematics, namely homotopy theory. The short citation for the Royal Swedish Academy of Sciences' "2020 Rolf Schock Prize in logic and philosophy"says that it was awarded to Per Martin-Löf (shared with Dag Prawitz) "for the creation of constructive type theory.'In a longer statement, the prize committee recalls that constructive type theory is "a formal language in which it is possible to express constructive mathematics"(...) "[which] also functions as a powerful programming language and has had an enormous impact in logic, computer science and, recently, mathematics."

By introducing a framework in which the formalisation of the logical notion of equality is done via the so-called "identity type", one is presented with the possibility for a surprising connection between term rewriting and geometric concepts such as path and homotopy. And indeed, Martin-Löf's type theory (MLTT) allows for making useful bridges between 
theory of computation, algebraic topology, logic, categories, and higher algebra, and a single concept seems to serve as a bridging bond: "path". Its impact in mathematics has been felt more strongly since the start of V. Voevodsky's program on the univalent foundations of mathematics around 2005, and one specific aspect which we would like to address here is the calculation of fundamental groups of surfaces. We know that calculation of homotopy groups is in general much more difficult than some of the other homotopy invariants learned in algebraic topology. Now, by using an alternative formulation of the "identity type"which provides an explicit formal account of "path", operationally understood as an invertible sequence of rewrites (such as Church's "conversion"), and interpreted as a homotopy, we seek to provide examples of calculations of fundamental groups of surfaces such as the circle, the torus, the 2-holed torus, the Klein bottle, and the real projective plane. These examples seem to bear witness to the impact of MLTT in mathematics by offering formal tools to calculate and prove fundamental groups, as well as allowing to make such calculations and proofs amenable to be dealt with by systems of formal mathematics and interactive theorem provers such as Coq, Lean, and similar ones.

\section{A topological application of labelled natural deduction}

In homotopy theory, the fundamental group is the one formed by all equivalence classes up to homotopy of paths (loops) starting from a point $x_{0}$ and also ending at $x_{0}$. Since we use computational paths as the syntactic counterpart of homotopic paths in type theory, we will use terms to formalize loops in a surface. Consider the type $S^{1}$ (circle), and let $x_{0}: S^{1}$ be a base term, and $x_{0}=x_{\alpha}$ be a computational path that starts and ends at $x_{0}$, going around the circle clockwise. We can then define this path as a base path, capable of generating any path in the circle, and denote it by loop $_{x_{0}}$. However, for simplicity, we can omit the $x_{0}$, but it is implied that loops will be made at the base point and denoted by loop ${ }^{1}$. Thus, the path (loop) formed by two turns based on $x_{0}$, around the circle in a clockwise direction, can be denoted by $l o o p^{2}$; a counterclockwise loop for $l o o p^{-1}$, in general, loop ${ }^{n}$ denotes the path formed by $n$ clockwise turns in the circle, based on $x_{0}$, with $n \in \mathbb{Z}$. If $n=0$ we can say that this is the homotopic path to the point and denote it by $l o o p^{0}$.

Now, imagine the path formed by three clockwise turns and two counterclockwise turns. This path is different from $l o o p^{1}$, but it is equivalent to it, that is, we can say that it is a rewrite of the computational path loop or $l o o p^{1}$, so it is relevant here to define a rewrite equivalence, and we can simply denote by $\left[l o o p^{n}\right]_{r w}$ every computational path that is equivalent to, or a rewrite of, the $l o o p^{n}$ path.

Definition 2.1 Let: (i) A be a type; (ii) $x_{0}$ : A a base point; (iii) $x_{0} \frac{=}{\alpha_{i}} x_{0}$, be a family of generator paths with $i \in I$; (iv) a family of relationships between the terms paths $\tau_{j}\left(x_{0} \underset{\alpha_{r}}{=} x_{0}, x_{0} \underset{\alpha_{s}}{=} x_{0}\right)$. We can define the structure $\Pi_{1}\left(A, x_{0}\right)$ as the set of terms $\alpha_{x_{0}}$, given by finite applications of $\tau, \sigma$, and $\rho$ in $\alpha_{i}$, modulo rw equality and modulo family of identity type terms $I d_{\tau_{j}}$.

Since each element in $\Pi_{1}\left(A, x_{0}\right)$ is a loop in $x_{0}$, we have:

Definition 2.2 Let $\left[\text { loop }^{n}\right]_{r w}$ be the path naturally obtained by the application of the path-axioms $\rho, \tau$ and $\sigma$ to the base path $x_{0} \underset{\text { loop }}{=} x_{0}$, where $n \in \mathbb{N}$. Particularly we can say: (i) $\left[\text { loop }^{0}\right]_{r w}=\left[\rho_{x_{0}}\right]_{r w}, n=0$; (ii) $\left[\text { loop }^{1}\right]_{r w}=[\text { loop }]_{r w}$; (iii) $\left[l_{\text {loop }}^{n}\right]_{r w}=$ $\tau\left(\left[\text { loop }^{n-1}\right]_{r w},\left[\text { loop }^{1}\right]_{r w}\right), n>0 ;($ iv $)\left[\text { loop }^{n}\right]_{r w}=\sigma\left(\left[\text { loop }^{-n}\right]_{r w}\right),-n>0$. 


\subsection{Fundamental group of the circle}

Definition 2.3 (The circle $S^{1}$ ) The circle is the type generated by: (i) A base point $x_{0}: S^{1}$; (ii) A base computational path $-x_{0} \underset{\text { loop }}{=} x_{0}: S^{1}$.

The first thing one should notice is that this definition does not use only the points of the type $S^{1}$, but also a base computational path called loop between those points. That is why it is called a higher inductive type. Our approach differs from the one developed in the HoTT book in that we do not need to simulate the path-space between those points via an encode-decode function, since we have paths in the syntax of the theory. (Even if the following are not new results, the point is that they are new proofs which are formalised in a way that the paths are explicitly given in the syntax.)

Theorem 2.1 ([Veras et al. 2019]) $\Pi_{1}\left(S^{1}, x_{0}\right) \simeq \mathbb{Z}$.

\subsection{Fundamental group of the torus}

Consider $\mathbb{T}^{2}$ as the surface known as Torus and the point $x_{0} \in \mathbb{T}^{2}$. We prove that the fundamental group of the torus is isomorphic to $\mathbb{Z} \times \mathbb{Z}$. Here we will also use Definition 2.1 with some simple adaptations. We work with paths up to $r w$-equality.

Theorem 2.2 ([Veras et al. 2019]) $\Pi_{1}\left(\mathbb{T}^{2}, x_{0}\right) \simeq \mathbb{Z} \times \mathbb{Z}$.

\subsection{Fundamental group of the real projective plane}

The real projective plane, denoted by $\mathbb{R P}^{2}$, is by definition the set of all straight lines that pass through the origin of space $\mathbb{R}^{3}$. We can define each of these lines by a position vector $v_{r}$, with $\left\|v_{r}\right\| \neq 0$, so we have that $\mathbb{R P}^{2}$ is a quotient space of $\mathbb{R}^{3}-(0,0)$ under the equivalence relation $v_{r} \sim \lambda v_{r}$ for scalars $\lambda \neq 0$. If we impose the condition that the vectors $\left\|v_{r}\right\|=1$ then $\mathbb{R P}^{2}$ is a quotient space $\mathbb{S}^{2}$ under the equivalence relation $v_{r} \sim-v_{r}$, the sphere with antipodal points identified, where $v_{r}$ is position vector.

Theorem 2.3 ([Veras et al. 2019]) $\Pi_{1}\left(\mathbb{R P} P^{2}\right) \simeq \mathbb{Z}_{2}$.

\section{Referências}

de Oliveira, A. G. (1995). Proof transformations for labelled natural deduction via term rewriting. Master's thesis, Informática, UFPE, Recife, Brazil, April 1995.

de Queiroz, R. J. G. B., de Oliveira, A. G., and Gabbay, D. M. (2011). The Functional Interpretation of Logical Deduction. World Scientific.

de Queiroz, R. J. G. B., de Oliveira, A. G., and Ramos, A. F. (2016). Propositional equality, identity types, and direct computational paths. Sth Am.J.Log., 2(2):245-96.

de Queiroz, R. J. G. B. and Gabbay, D. M. (1994). Equality in labelled deductive systems and the functional interpretation of propositional equality. In Proc. 9th Amsterdam Coll., pages 547-565. ILLC/Dept of Phil. Univ Amsterdam.

Martin-Löf, P. (1975). An intuitionistic theory of types: Predicative part. In Rose, H. and Shepherdson, J., editors, Proc. Logic Colloquium '73, pages 73 - 118. Elsevier.

Ramos, A. F., de Queiroz, R. J. G. B., and de Oliveira, A. G. (2017). On the identity type as the type of computational paths. Logic Journal of IGPL, 25(4):562-584. 
Veras, T. M. L., Ramos, A. F., de Queiroz, R. J. G. B., and de Oliveira, A. G. (2019). A topological application of labelled natural deduction. arXiv:1906.09105.

Veras, T. M. L., Ramos, A. F., de Queiroz, R. J. G. B., Silva, T. D. O., and de Oliveira, A. G. (2020). Computational paths - a weak groupoid. arXiv: 2007.07769. 